\title{
Collateral or Utility Penalties?
}

\author{
Wilfredo L. Maldonado ${ }^{\dagger}$ \\ Graduate School in Economics, Catholic University of Brasília \\ SGAN 916, Módulo B, CEP 70790-160 \\ Brasília DF, BRAZIL \\ Jaime Orrillo \\ Graduate School in Economics, Catholic University of Brasília \\ SGAN 916, Módulo B, CEP 70790-160 \\ Brasília DF, BRAZIL
}

\begin{abstract}
In a two-period economy with incomplete markets and possibility of default we consider the two classical ways to enforce the honor of financial commitments: by using utility penalties and by using collateral requirements that borrowers have to fulfill. Firstly, we prove that any equilibrium in an economy with collateral requirements is also equilibrium in a non-collateralized economy where each agent is penalized (rewarded) in his utility if his delivery rate is lower (greater) than the payment rate of the financial market. Secondly, we prove the converse: any equilibrium in an economy with utility penalties is also equilibrium in a collateralized economy. For this to be true the payoff function and initial endowments of the agents must be modified in a quite natural way. Finally, we prove that the equilibrium in the economy with collateral requirements attains the same welfare as in the new economy with utility penalties.
\end{abstract}

Keywords: Incomplete markets; Exogenous collateral; Utility penalties JEL classification: D52

\footnotetext{
$\dagger$ I would like to thank CNPq of Brazil for financial support 305317/2003-2 and Edital Universal 471899/2003-8.

* Corresponding author, e-mail: orrllo@ pos.ucb.br. We acknowledge the comments of Mário Páscoa at the Workshop in Public Economics and Economic Theory 2004.
} 


\section{Introduction}

One of the concerns of the modern general equilibrium theory with incomplete markets (GEI) is the possibility of agents who do not honor their financial commitments. Since non-negligible default is observed in the real world, it is necessary to use a realistic model to capture the possibility of its occurrence. This is done in order to analyze the implications of default and evaluate policies which avoid financial crashes or loss of efficiency.

In Dubey, Geanakoplos and Shubik (2004) agents are allowed to default on financial debts but each unit of financial debt that is not paid is penalized directly in the utility function. Thus each agent has a payoff function which depends on the private consumption and the amount of non-paid financial debt. On the other hand, Geanakoplos and Zame (2002) model the possibility of default by allowing the borrowers to deliver a previously constituted collateral if its value is lower than the value of the financial debt. In both cases the default is strategic and chosen by the agent.

The utility penalty parameters used in the former approach are usually related to the loss of credibility of the defaulter in future periods, which restricts his access to the credit market in those periods. The parameters are also interpreted as a sort of non-economic punishment that the agent suffers when the debt is not completely paid. From the theoretical point of view, the possibility of default using utility penalties improves the efficiency of the equilibrium allocations as proved by Zame (1993). The main advantage of using utility penalties is its analytic treatment in applied models (see for example Goodhart, Sunirand and Tsomocos (2003a, b)).

The use of collateral requirements provides in some cases a more realistic alternative to model the possibility of default. If the borrower desires a loan he has to constitute a collateral bundle (that can be used by him or by the lender) which may be confiscated if the debt is not paid. In addition to being a more realistic device than utility penalties, their inclusion in models of infinite horizon, as shown by Araujo, Páscoa and Torres- 
Martinez (2002) and Orrillo (2002), avoids Ponzi schemes. To see other benefits of collateral in economies with infinite horizons and defaults, we refer to Kubler and Scemeders (2002). However, it is interesting to note that some kinds of loans are not backed by collateral (for example, loans related to sovereign debt or credit cards). Besides the benefits of the collateral mentioned above, there are economic and analytic complications to the policy maker if he decides to use this collateral framework in applied models.

The question is: given an equilibrium (prices, allocations of consumption and portfolio and decision of default), is one of these alternatives better than the other to support/explain it? In this paper we show that both approaches are equivalent (in the sense described below) to explain any equilibrium. Specifically, suppose that we have an equilibrium in an economy with collateral requirements. We can then find a system of penalty rates such that the economy with these utility penalties supports the same prices and allocations as equilibrium. In this new economy agents must be punished (rewarded) if they deliver less (more) than the average delivery of the financial market. Reciprocally, any equilibrium in an economy with utility penalties is also an equilibrium in a collateralized economy. For this to be true we need to modify the payoff function and initial endowments of each agent in an appropriate way.

The implications of the results above are clear. If the number of commodities in a collateralized economy is greater than the number of states, then we can replace the enforcement mechanism by one which uses utility penalties. This may be an important simplification for applied economists, since the calibration of utility penalty parameters is easier than the processing of data to determine the collateral structure. On the other hand, moving from a utility penalty system to a collateral one allows us to evaluate the impacts of structural changes on the mechanisms which enforce the honoring of financial commitments. This is analyzed in detail in Subsection 3.2.

Once stated the equivalence above, we discuss its welfare implications. If one passes from the economy with collateral requirements to the economy with utility penalties, the 
equilibrium allocation maintains the same utility profile of the agents. This means that it does not matter which mechanism enforces the honor of commitments - the agents end up with the same utility in equilibrium. However, when we pass from the economy with utility penalties to the economy with collateral requirements the social welfare is not comparable, in general. Although we cannot maintain the utility of the agents in equilibrium, as in the first case, we prove that the cost/benefit of passing from a penalty system to a collateralized one is equal to the variation of the individual payoff.

The paper is organized as follows: Section 2 describes the simple two-period setting for the GEI and the two forms to enforce the financial commitments, namely utility penalties and collateral requirements. Section 3 presents the main results, which establish the equivalence between the two mechanisms. We also analyze the welfare implications of this equivalence. Section 4 is devoted to some concluding remarks, and all the proofs are given in the appendix.

\section{The Basic GEI Model with Possibility of Default}

We begin by describing the two classical models which represent situations where agents may non-honor their financial commitments. The economy extends over two periods and uncertainty exists only in the second period. There exist $L$ physical goods in each period which are traded in spot markets. In the sequel we will use the same letter to denote either the set or its cardinality. The uncertainty in the second period is described by the set of states $S=\{1, \ldots, S\}$. There exist $J$ financial assets, each one offering a contingent real return given by $A_{s}^{j} \in R_{+}^{L}$. Markets are incomplete $(S>J)$ and since goods can be durable, the depreciation in state $s$ is described by the matrix $Y_{s} \in R_{+}^{L \times L}$.

There exist $H$ agents, each one characterized by his consumption set $X^{h} \subset R_{+}^{L(S+1)}$, utility function on private consumption $u^{h}: X^{h} \rightarrow R$ and initial endowments $w^{h} \in X^{h}$. A consumption plan for agent $h$ is denoted by $x=\left(x_{0}, x_{1}, \ldots, x_{S}\right) \in X^{h}$. Analogously, the

purchases and sales of financial assets are denoted by $\theta=\left(\theta_{1}, \ldots, \theta_{J}\right) \in R_{+}^{J}$ and 
$\varphi=\left(\varphi_{1}, \ldots, \varphi_{J}\right) \in R_{+}^{J}$ respectively. The following hypothesis will be assumed in all theorems below:

Hypothesis: For each $h, u^{h}$ is a continuous and concave function on its domain and $w^{h}>0$.

The commodity prices are denoted by $p_{0} \in R_{+}^{L}$ for goods in the first period and $p_{s} \in R_{+}^{L}$ for goods in the second period if state $s$ occurs. Asset prices are denoted by $\pi \in R_{+}^{J}$ and they are traded in the first period.

If agent $h$ sells $\varphi_{j}$ units of asset $j$ then his debt for the second period will be $p_{s} A_{s}^{j} \varphi_{j}$ and since we are allowing the possibility of default on that debt, it is necessary to define mechanisms to enforce the repayment of (at least part of) that debt.

\subsection{The Economy with Exogenous Collateral}

In this setting each asset $j \in J$ is backed by a collateral bundle $C_{j} \in R_{+}^{L}$ in the following sense: If an individual wants to sell $\varphi_{j}$ units of asset $j$ then he must buy $C_{j} \varphi_{j}$ units of goods (that can be used by himself) that will be confiscated if the financial debt $p_{s} A_{s}^{j} \varphi_{j}$ is not paid. Thus, it is publicly known that each unit of asset $j$ will deliver in state $s: d_{s}^{j}=\operatorname{Min}\left\{p_{s} A_{s}^{j}, p_{s} Y_{s} C_{j}\right\}$. Therefore the economy with incomplete markets, possibility of financial default and collateral requirements is defined by:

$$
\varepsilon_{C}=\left\{\left(X^{h}, u^{h}, w^{h}\right)_{h \in H},\left(\left(A_{s}^{j}\right)_{s \in S}, C_{j}\right)_{j \in J},\left(Y_{s}\right)_{s \in S}\right\}
$$

The definition above assumes that the preferences of individuals are defined on private commodity consumption. However, more general settings can be considered which allow preferences to also be defined on the portfolio plans of the individual. In these cases we can consider a payoff function $V^{h}: X^{h} \times R_{+}^{2 J} \rightarrow R$ for agent $h$. In the particular case above $V^{h}(x, \theta, \varphi)=u^{h}\left(x_{0}+C \varphi,\left(x_{s}\right)\right)$. 
An equilibrium for $\varepsilon_{C}$ is a vector of prices $(p, \pi) \in R_{+}^{L \times(S+1)} \times R_{+}^{J}$ and consumptioninvestment allocation $\left(x^{h}, \theta^{h}, \varphi^{h}\right) \in X^{h} \times R_{+}^{2 J}$ for each $h \in H$ such that:

1. For each $h \in H,\left(x^{h}, \theta^{h}, \varphi^{h}\right)$ maximizes $u^{h}\left(x_{0}+C \varphi,\left(x_{s}\right)\right)$ on the set of consumption-investment plans $(x, \theta, \varphi) \in X^{h} \times R_{+}^{2 J}$ that satisfy:

$$
\begin{gathered}
p_{0} x_{0}+\pi \theta+p_{0} C \varphi \leq p_{0} w_{0}^{h}+\pi \varphi \\
p_{s} x_{s}+\sum_{j} d_{s}^{j} \varphi_{j} \leq p_{s} w_{s}^{h}+\sum_{j} d_{s}^{j} \theta_{j}+\sum_{j} p_{s} Y_{s} C_{j} \varphi_{j}+p_{s} Y_{s} x_{0} ; s \in S
\end{gathered}
$$

2. Markets clear:

$$
\begin{gathered}
\sum_{h}\left(x_{0}^{h}+C \varphi^{h}\right)=\sum_{h} w_{0}^{h} \\
\sum_{h} x_{s}^{h}=\sum_{h}\left[w_{s}^{h}+Y_{s}\left(x_{0}^{h}+C \varphi^{h}\right)\right] s \in S \\
\sum_{h} \theta^{h}=\sum_{h} \varphi^{h}
\end{gathered}
$$

\subsection{The Economy with Utility Penalties}

The other form to enforce the repayment of the debt is assuming the existence of a penalty directly applied to the utility of consumption. In this way, if agent $h$ decides to deliver a part of his debt given by $D_{s}^{j} \in\left[0, p_{s} A_{s}^{j} \varphi_{j}\right]$, then his total payoff is given by $V^{h}(x, \varphi, D)=u^{h}\left(x_{0},\left(x_{s}\right)\right)-\sum_{j, s} \lambda_{s}^{h j}\left[p_{s} A_{s}^{j} \varphi_{j}-D_{s}^{j}\right]^{+}$. As in the model described in section 2.1, the payoff function may assume more general forms than the quasi-linear form considered here.

Since the penalty is on the non-paid debt, large amounts of short sales may occur, therefore a bounded short sale $v \in R_{+}^{J}$ of financial assets must be considered. Therefore 
the economy with incomplete markets, possibility of financial default and utility penalties is defined by:

$$
\varepsilon_{P}=\left\{\left(X^{h}, u^{h}, w^{h}\right)_{h \in H},\left(\left(A_{s}^{j}, \lambda_{s}^{j}\right)_{s \in S} \in J, v\right),\left(Y_{s}\right)_{s \in S}\right\}^{1}
$$

Since borrowers may default, lenders are aware that they will not receive the total return of their investments. Therefore they publicly assume that the rate of repayment of asset $j$ in state $s$ is $t_{s}^{j} \in[0,1]$, which means that if a lender buys $\theta_{j}$ units of asset $j$ then the return of this investment is given by $t_{s}^{j} p_{s} A_{s}^{j} \theta_{j}$.

An equilibrium for the economy $\varepsilon_{P}$ is a vector of prices $(p, \pi) \in R_{+}^{L(S+1)} \times R_{+}^{J}$, repayment rates $t \in[0,1]^{S \times J}$ and for each $h$ a consumption-investment-delivery plan $\left(x^{h}, \theta^{h}, \varphi^{h}, D^{h}\right) \in X^{h} \times R_{+}^{2 J} \times R_{+}^{S \times J}$ such that:

1. For each $h,\left(x^{h}, \theta^{h}, \varphi^{h}, D^{h}\right)$ maximizes $V^{h}(x, \varphi, D)$ on the set of consumption investment-delivery plans $(x, \theta, \varphi, D) \in X^{h} \times R_{+}^{2 J} \times R_{+}^{S \times J}$ that satisfies:

$$
\begin{gathered}
p_{0} x_{0}+\pi \theta \leq p_{0} w_{0}^{h}+\pi \varphi \\
p_{s} x_{s}+\sum_{j=1}^{J} D_{s}^{j} \leq p_{s} w_{s}^{h}+\sum_{j=1}^{J} t_{s}^{j} p_{s} A_{s}^{j} \theta_{j}+p_{s} Y_{s} x_{0} ; s \in S
\end{gathered}
$$

2. Markets clear:

$$
\begin{gathered}
\sum_{h} x_{0}^{h}=\sum_{h} w_{0}^{h} \\
\sum_{h} x_{s}^{h}=\sum_{h}\left[w_{s}^{h}+Y_{s} x_{0}^{h}\right] \quad s \in S \\
\sum_{h} \theta^{h}=\sum_{h} \varphi^{h}
\end{gathered}
$$

3. The payment rate is correctly anticipated (rational expectations hypothesis):

$$
t_{s}^{j}=\frac{\sum_{h=1}^{H} D_{s}^{j h}}{\sum_{h=1}^{H} p_{s} A_{s} \varphi_{j}^{h}}, \text { provided that } \sum_{h=1}^{H} \varphi_{j}^{h} \neq 0
$$

\footnotetext{
${ }^{1}$ To simplify notation we delete the $h$-index from the penalty rates.
} 


\section{Results}

\subsection{From Collateral to Utility Penalties}

In this section we will present the main results of the paper. The first one states that any equilibrium in an economy with collateral requirements can be found in an economy with utility penalties with the same initial endowments, if the payoff functions of individuals are modified conveniently.

Theorem 3.1. Let $\left(p, \pi,\left(x^{h}, \theta^{h}, \varphi^{h}\right)_{h \in H}\right)$ be an equilibrium of the economy with collateral requirements $\varepsilon_{C}=\left\{\left(X^{h}, u^{h}, w^{h}\right)_{h \in H},\left(\left(A_{s}^{j}\right)_{s \in S}, C_{j}\right)_{j \in J},\left(Y_{s}\right)_{s \in S}\right\}$. If we define the repayment rate $t \in[0,1]^{S \times J}$, individual delivery decisions $D^{h} \in R_{+}^{S \times J}$ and payoff functions $\tilde{V}^{h}(x, \varphi, D)$ as:

$$
\begin{gathered}
t_{s}^{j}=\frac{d_{s}^{j}}{p_{s} A_{s}^{j}}=\operatorname{Min}\left\{1, \frac{p_{s} Y_{s} C_{j}}{p_{s} A_{s}^{j}}\right\} ; \quad D_{s}^{h j}=d_{s}^{j} \varphi_{j}^{h} \text { and } \\
\tilde{V}^{h}(x, \varphi, D)=u^{h}(x)-\sum_{s, j} \beta_{s}^{h}\left[t_{s}^{j} p_{s} A_{s}^{j} \varphi_{j}-D_{s}^{j}\right]
\end{gathered}
$$

(where $\beta_{s}^{h}$ is the Lagrange multiplier of the s-budget constrain of $h$ in $\varepsilon_{C}$ ) then $\left(p, \pi, t,\left(x_{0}^{h}+C \varphi^{h}, x_{s}^{h}, \theta^{h}, \varphi^{h}, D^{h}\right)_{h \in H}\right)$ is an equilibrium for the economy with utility penalties $\varepsilon_{P}=\left\{\left(X^{h}, \tilde{V}^{h}, w^{h}\right)_{h \in H},\left(\left(A_{s}^{j}\right)_{s \in S} j \in J, v\right),\left(Y_{s}\right)_{s \in S}\right\}$ for some bounded short sales $v$.

It is important to note the following:

1.- In the economy $\varepsilon_{C}$ agents must purchase durable goods which serve as collateral for each asset sold. Since in the economy $\varepsilon_{P}$ it is not necessary, the total consumption in the first period must remain as $x_{0}^{h}+C \varphi^{h}$.

2.- Initial endowments, asset returns structure and depreciation rates remain the same. This is an important fact for applied economists because they can choose the simplest model from the same initial data.

3.- Prices and allocations are the same in equilibrium for both economies. 
4.- The payoff functions of the agents can be read as follows: in the new economy, an agent is punished if his delivery rate $\left(D_{s}^{j} /\left(p_{s} A_{s}^{j} \varphi_{j}\right)\right)$ is lower than the payment rate $t_{s}^{j}$ of the economy and he is rewarded in the other case. Also, if $k_{s}^{j}$ is the rate of default in asset $j$ if state $s$ occurs, then the payoff function can be re-written as:

$$
V^{h}(x, \varphi, D)=u^{h}(x)-\sum_{s, j} \beta_{s}^{h} t_{s}^{j}\left[p_{s} A_{s}^{j} \varphi_{j}-D_{s}^{j}\right]+\sum_{s, j} \beta_{s}^{j} k_{s}^{j} D_{s}^{j} .
$$

This is the same Dubey, Geanakoplos and Shubik (2004) payoff function (with a personalized default penalty $\lambda_{s}^{h j}=\beta_{s}^{h} t_{s}^{j}$ ) plus a term which is proportional to the market default rate. This last term encourages the delivery of the debt.

It is worth noting that Theorem 3.1 proposes the translation of a collateralized equilibrium to equilibrium in an economy with utility penalties. The former is a physical enforcement mechanism whereas the other is a subjective (and probably non-observed) enforcement mechanism. In spite of that translation which modifies the payoff function of individuals, they end up with the same private welfare. This is stated in the following corollary.

Corollary 3.2. With the notation of Theorem 3.1 we can conclude that:

$$
\tilde{V}^{h}\left(x_{0}^{h}+C \varphi^{h},\left(x_{s}^{h}\right), \varphi^{h}, D^{h}\right)=u^{h}\left(x_{0}^{h}+C \varphi^{h},\left(x_{s}\right)\right) ; \quad \forall h \in H .
$$

This means that for the economies $\varepsilon_{C}$ and $\varepsilon_{P}$ of Theorem 3.1 the type of enforcement mechanism (collateral requirements or utility penalties) does not matter, from the social point of view. The individuals will end up with the same welfare.

\subsection{From Utility Penalties to Collateral}

In Theorem 3.1 we can see that the market default rate is the same in the two economies. Furthermore the delivery per unit of each asset sold $\left(D_{s}^{h j} / \varphi_{j}^{h}\right)$ is the same for all individuals. It is a particular property in an economy with exogenous collateral requirements. The delivery per unit of each asset sold may vary from individual to 
individual in an economy with utility penalties. In this case it is difficult to define a collateral structure that supports the same default rate in equilibrium.

To state the converse of Theorem 3.1, we are going to introduce the following notation. Let $\left(p, \pi, t,\left(x^{h}, \theta^{h}, \varphi^{h}, D^{h}\right)_{h \in H}\right)$ be an equilibrium of the economy with utility penalties $\varepsilon_{P}=\left\{\left(X^{h}, u^{h}, w^{h}\right)_{h \in H},\left(\left(A_{s}^{j}, \lambda_{s}^{j}\right)_{s \in S} \in J, v\right),\left(Y_{s}\right)_{s \in S}\right\}$ and $\rho_{s}^{h j}=D_{s}^{h j} /\left(p_{s} A_{s}^{j} \varphi_{j}^{h}\right)$ (if the denominator is zero, define $\left.\rho_{s}^{h j}=0\right)$. For any given collateral system $C \in R_{+}^{L \times J}$ define $d_{s}^{j}=\operatorname{Min}\left\{p_{s} A_{s}^{j}, p_{s} Y_{s} C_{j}\right\}$. The monetary return of the agent $h$ portfolio in state $s$ is:

$$
\begin{gathered}
r_{s}^{h}=\sum_{j \in J}\left(t_{s}^{j} p_{s} A_{s}^{j} \theta_{j}^{h}-\rho_{s}^{h j} p_{s} A_{s}^{j} \varphi_{j}^{h}\right), \quad \text { (in the economy with utility penalties) } \\
\tilde{r}_{s}^{h}=\sum_{j \in J} d_{s}^{j}\left(\theta_{j}^{h}-\varphi_{j}^{h}\right), \quad \text { (in theeconomy with collateralrequirements) }
\end{gathered}
$$

Analogously, the present value (in utility terms) of the portfolio $(\theta, \varphi) \in R_{+}^{2 J}$ is:

$$
\begin{gathered}
R^{h}(\theta, \varphi)=\sum_{j \in J, s \in S} \alpha_{s}^{h}\left(t_{s}^{j} p_{s} A_{s}^{j} \theta_{j}-\rho_{s}^{h j} p_{s} A_{s}^{j} \varphi_{j}\right), \quad \text { (in the economy with utility penalties) } \\
\tilde{R}^{h}(\theta, \varphi)=\sum_{j \in J, s \in S} \alpha_{s}^{h} d_{s}^{j}\left(\theta_{j}-\varphi_{j}\right), \quad \text { (in the economy with collateralrequirements) }
\end{gathered}
$$

where $\alpha_{s}^{h} \in R_{+}$is the Lagrange multiplier of the $s$-budget constrain of agent $h$ in $\varepsilon_{P}$.

Finally, let us define the set $J_{s}=\left\{j \in J / p_{s} A_{s}^{j} \leq p_{s} Y_{s} C_{j}\right\}$ (the set of all assets with honored promises in state $s$ ).

With these notations we have the following theorem.

Theorem 3.3. Let $\left(p, \pi, t,\left(x^{h}, \theta^{h}, \varphi^{h}, D^{h}\right)_{h \in H}\right)$ be an equilibrium of the economy with utility penalties $\varepsilon_{P}=\left\{\left(X^{h}, u^{h}, w^{h}\right)_{h \in H},\left(\left(A_{s}^{j}, \lambda_{s}^{j}\right)_{s \in S} \in J, v\right),\left(Y_{s}\right)_{s \in S}\right\}$ with $D^{h} \gg 0$ for all $h \in H$. Then for any collateral system $C \in R_{++}^{J \times L}$, the vector of prices and allocations $\left(p, \pi,\left(x^{h}, \theta^{h}, \varphi^{h}\right)_{h \in H}\right)$ is an equilibrium of the economy with collateral requirements $\varepsilon_{C}=\left\{\left(X^{h}, \tilde{V}^{h}, \tilde{w}^{h}\right)_{h \in H},\left(\left(A_{s}^{j}\right)_{s \in S}, C_{j}\right)_{j \in J},\left(Y_{s}\right)_{s \in S}\right\}$ and bounded short sales $v$, where: 


$$
\begin{gathered}
\widetilde{w}_{s}^{h}=w_{s}^{h}+\sum_{j} A_{s}^{j}\left(t_{s}^{j} \theta_{j}^{h}-\rho_{s}^{h j} \varphi_{j}^{h}\right)-\sum_{j \in J_{s}} A_{s}^{j}\left(\theta_{j}^{h}-\varphi_{j}^{h}\right)-\sum_{j \notin J_{s}} Y_{s} C_{j}\left(\theta_{j}^{h}-\varphi_{j}^{h}\right)-Y_{s} C \varphi^{h} \\
\widetilde{w}_{0}^{h}=w_{0}^{h}+C \varphi^{h}
\end{gathered}
$$

and:

$$
\tilde{V}^{h}(x, \varphi, \theta)=u^{h}(x)+\sum_{s, j} \alpha_{s}^{h}\left(t_{s}^{j} p_{s} A_{s}^{j} \theta_{j}-p_{s} A_{s}^{j} \varphi_{j}\right)-\tilde{R}^{h}(\theta, \varphi)+\alpha_{0}^{h}\left[p_{0}-\sum_{s \in S} \frac{\alpha_{s}^{h}}{\alpha_{0}^{h}} p_{s} Y_{s}\right] C \varphi .
$$

It is easy to verify that $p_{s} \tilde{w}_{s}^{h}+\tilde{r}_{s}^{h}+p_{s} Y_{s} C \varphi^{h}=p_{s} w_{s}^{h}+r_{s}^{h}>0$. Therefore, although $\widetilde{w}_{s}^{h}$ may not be in $R_{+}^{L}$, the total wealth (financial and non-financial) is strictly positive at least for one consumption-investment plan, so the budget constraint has a nonempty interior in the new collateralized economy.

The payoff function of agent $h$ in the new economy of Theorem 3.3 has a quite intuitive interpretation. The last term $\alpha_{0}^{h}\left|p_{0}-\sum_{s}\left(\alpha_{s}^{h} / \alpha_{0}^{h}\right) p_{s} Y_{s}\right| C \varphi$ is the net value of the collateral bundle in utility units. Since that collateral requirement did not exist in $\varepsilon_{P}$, this term has to be added to the utility of consumption. The term $\widetilde{R}^{h}(\theta, \varphi)$ is the net financial return in a collateralized economy (in utility units). It has to be subtracted from the consumption utility in order to compensate its inclusion in the new budget constrain. Finally, the second term in the new payoff function can be written as:

$$
\sum_{s, j} \alpha_{s}^{h}\left(t_{s}^{j} p_{s} A_{s}^{j} \theta_{j}-D_{s}^{j}\right)-\sum_{s, j} \alpha_{s}^{h}\left(p_{s} A_{s}^{j} \varphi_{j}-D_{s}^{j}\right)
$$

Here the first term corresponds to the net financial return in an economy with utility penalties. Since this return is dropped out from the budget constraint, we have to compensate that effect in the utility of consumption. The second term represents the value of the default given by $h$. It must be subtracted from the utility of consumption because that term corresponds to an implicit gain that agent $h$ had in the former budget constraint. We can summarize the composition of the new payoff function in the following diagram. 


$$
\begin{aligned}
& \begin{array}{|c|c|c|}
\hline \begin{array}{c}
\text { The new } \\
\text { payoff function }
\end{array} & =\begin{array}{c}
\text { Utility of } \\
\text { consumption }
\end{array}
\end{array}+\begin{array}{c}
\begin{array}{c}
\text { Utility of eliminated } \\
\text { termsin the budget } \\
\text { constraint }
\end{array} \\
\text { constraint }
\end{array} \\
& +\begin{array}{|c|}
\hline \begin{array}{c}
\text { Net effect of } \\
\text { collateral implementation }
\end{array} \\
\text { fet effect of dropping out } \\
\text { from utility penalties system }
\end{array}
\end{aligned}
$$

Theorem 3.3 is important not only because it allows us to see an equilibrium in an economy with utility penalties as a collateralized equilibrium. It also provides a very intuitive way to implement a collateral system by a central planner (CP). Suppose that a $\mathrm{CP}$ wants the implementation of a collateral system $C$ in an economy with utility penalties. It must be done maintaining the same equilibrium. The $\mathrm{CP}$ should then execute the following steps:

i) In $t=0$ the CP must lend $C \varphi^{h}$ to individual $h$. This is in order to preserve the initial consumption and to allow the purchase of collateral. It implies that the new initial endowment will be $\widetilde{w}_{0}^{h}=w_{0}^{h}+C \varphi^{h}$.

ii) In $t=1$ the CP transfers the amount $r_{s}^{h}$ to individual $h$ in the state $s$. This is done because the $\mathrm{CP}$ must compensate individuals for having past from a utility penalty system to a collateral system. So the monetary return of the former system has to be paid. Note that $\sum_{h \in H} r_{s}^{h}=0$. Therefore, it is a lump-sum transfer among individuals.

iii) In $t=1$ the $\mathrm{CP}$ receives from $h$ the value of the depreciated collateral (received in i)) in state $s$, namely $p_{s} Y_{s} C \varphi^{h}$.

iv) Finally, in $t=1 \mathrm{CP}$ receives the amount $\tilde{r}_{s}^{h}$ from individual $h$ in state $s$. This is the payment that has to be made to implement the new system of collateral. Again, this is a lump-sum transfer since $\sum_{h \in H} \tilde{r}_{s}^{h}=0$.

Observe that ii), iii) and iv) imply that the new initial wealth in $t=1$ of individual $h$ in the state $s$ is $p_{s} w_{s}^{h}+r_{s}^{h}-\tilde{r}_{s}^{h}-p_{s} Y_{s} C \varphi^{h}$ which in fact is equal to $p_{s} \tilde{w}_{s}^{h}$.

It is also worth noting that in the equilibrium agent $h$ attains the following payoff: 


$$
\begin{aligned}
\tilde{V}^{h}\left(x^{h}, \varphi^{h}, \theta^{h}\right)= & u^{h}\left(x^{h}\right)-\sum_{s, j} \lambda_{s}^{j}\left[p_{s} A_{s}^{j} \varphi_{j}^{h}-D_{s}^{h j}\right]+R^{h}\left(\theta^{h}, \varphi^{h}\right)-\tilde{R}^{h}\left(\theta^{h}, \varphi^{h}\right) \\
& +\alpha_{0}^{h}\left[p_{0}-\sum_{s \in S} \frac{\alpha_{s}^{h}}{\alpha_{0}^{h}} p_{s} Y_{s}\right] C \varphi^{h} .
\end{aligned}
$$

We can interpret this payoff from remarks i) to iv). In terms of utility, i) and iii) imply that the payoff of agent $h$ is increased in $\alpha_{0}^{h}\left[p_{0}-\sum_{s}\left(\alpha_{s}^{h} / \alpha_{0}^{h}\right) p_{s} Y_{s}\right] C \varphi^{h}$. Furthermore, the transfer given in ii) increases the payoff function in $R^{h}\left(\theta^{h}, \varphi^{h}\right)$ and the payment made in iii) decreases the payoff in $\tilde{R}^{h}\left(\theta^{h}, \varphi^{h}\right)$. With all these modifications, the new payoff of agent $h$ becomes $\tilde{V}^{h}\left(x^{h}, \theta^{h}, \varphi^{h}\right)$.

When we pass from $\varepsilon_{P}$ to $\varepsilon_{C}$ an analogous result to Corollary 3.2 cannot be obtained. Since it is not true that a borrower (lender) delivers (receives) the same amount in the new economy, he can improve or not his individual welfare. Additionally, the new payoff includes the net personal utility of the collateral borrowed from the CP. Nevertheless, the net value (in $h$-utility units) of passing from an economy with utility penalties to a collateralized economy is given by:

$$
R^{h}\left(\theta^{h}, \varphi^{h}\right)-\tilde{R}^{h}\left(\theta^{h}, \varphi^{h}\right)+\alpha_{0}^{h}\left[p_{0}-\sum_{s}\left(\frac{\alpha_{0}^{h}}{\alpha_{0}^{h}}\right) p_{s} Y_{s}\right] C \varphi^{h},
$$

which is exactly $\tilde{V}^{h}-V^{h}$. It means that the cost/benefit of implementing a collateral system in an economy with utility penalties is equal to the individual's $h$ payoff change.

For the sake of completeness, we state a theorem which is similar to Theorem 3.3 but includes the case where agents may deliver nothing in the equilibrium of $\varepsilon_{P}$. Again, let $\left(p, \pi, t,\left(x^{h}, \theta^{h}, \varphi^{h}, D^{h}\right)_{h \in H}\right)$ be an equilibrium of the economy with utility penalties $\varepsilon_{P}=\left\{\left(X^{h}, u^{h}, w^{h}\right)_{h \in H},\left(\left(A_{s}^{j}, \lambda_{s}^{j}\right)_{s \in S}{ }_{j \in J}, v\right),\left(Y_{s}\right)_{s \in S}\right\}$ and for each $h \in H, j \in J, s \in S$ and $n$ (an integer greater than one) let us define the function $\phi_{s n}^{h j}$ on the interval $[0, v]$ as shown in figure 1: 


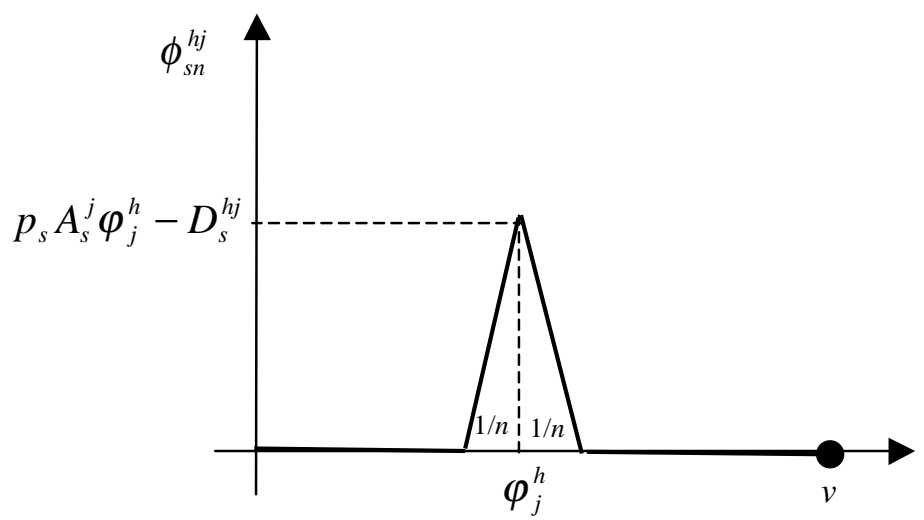

Figure 1

The $\phi_{s n}^{h j}$ function may be interpreted as a default strategy of the individual $h$. The net marginal utility for defaulting by using the strategy $\phi_{s n}^{h j}$ is given by:

$$
N_{n}(\varphi)=\sum_{s, j}\left(\alpha_{s}^{h}-\lambda_{s}^{j}\right) \phi_{s i}^{h j}\left(\varphi_{j}\right)
$$

We can observe that $N_{n}$ is zero outside of the set $\left[\varphi_{j}^{h}-(1 / n), \varphi_{j}^{h}+(1 / n)\right]^{J}$. With this notation we can state our last theorem.

Theorem 3.4. Let $\left(p, \pi, t,\left(x^{h}, \theta^{h}, \varphi^{h}, D^{h}\right)_{h \in H}\right)$ be an equilibrium of the economy with utility penalties $\varepsilon_{P}=\left\{\left(X^{h}, u^{h}, w^{h}\right)_{h \in H},\left(\left(A_{s}^{j}, \lambda_{s}^{j}\right)_{s \in S}, j \in J\right),\left(Y_{s}\right)_{s \in S}\right\}$. Then for each integer number $n \geq 1$ and each collateral system $C \in R_{++}^{J \times L}$, the prices and allocations $\left(p, \pi,\left(x^{h}, \theta^{h}, \varphi^{h}\right)_{h \in H}\right)$ is an equilibrium of the economy with collateral requirements $\varepsilon_{C, n}=\left\{\left(X^{h}, \tilde{V}_{n}^{h}, \tilde{w}^{h}\right)_{h \in H},\left(\left(A_{s}^{j}\right)_{s \in S}, C_{j}\right)_{j \in J},\left(Y_{s}\right)_{s \in S}\right\}$ and bounded short sales $v$, where:

$$
\begin{gathered}
\widetilde{w}_{s}^{h}=w_{s}^{h}+\sum_{j} A_{s}^{j}\left(t_{s}^{j} \theta_{j}^{h}-\rho_{s}^{h j} \varphi_{j}^{h}\right)-\sum_{j \in J_{s}} A_{s}^{j}\left(\theta_{j}^{h}-\varphi_{j}^{h}\right)-\sum_{j \notin J_{s}} Y_{s} C_{j}\left(\theta_{j}^{h}-\varphi_{j}^{h}\right)-Y_{s} C \varphi^{h} \\
\widetilde{w}_{0}^{h}=w_{0}^{h}+C \varphi^{h}
\end{gathered}
$$

and:

$$
\begin{aligned}
\tilde{V}_{n}^{h}(x, \varphi, \theta)= & u^{h}(x)+\sum_{s, j} \alpha_{s}^{h}\left(t_{s}^{j} p_{s} A_{s}^{j} \theta_{j}-p_{s} A_{s}^{j} \varphi_{j}\right)-\tilde{R}^{h}(\theta, \varphi)+\alpha_{0}^{h}\left[p_{0}-\sum_{s \in S} \frac{\alpha_{s}^{h}}{\alpha_{0}^{h}} p_{s} Y_{s}\right] C \varphi \\
& +N_{n}(\varphi) .
\end{aligned}
$$


We can observe that the payoff function of Theorem 3.4 coincides with that of Theorem 3.3 except in the set $\left[\varphi_{j}^{h}-(1 / n), \varphi_{j}^{h}+(1 / n)\right]^{J}$, which decreases as $n \rightarrow+\infty$.

All the analysis done after Theorem 3.3, with respect to the implementation of a collateral system in $\varepsilon_{P}$ and its individual cost/benefit, is also valid for Theorem 3.4.

\section{Concluding Remarks}

In the literature of general equilibrium theory with incomplete markets and possibility of default, the issue concerning the choice of the mechanism to enforce financial commitments is always discussed. From the theoretical point of view the use of collateral requirements seems more reasonable. However, the use of utility penalties which represent either exclusion from the credit markets in future periods or non-economic punishments has been well received, especially by applied economists.

In this paper we show how these two structures can be compatibilized in order to explain a specific equilibrium. More precisely, if we consider an equilibrium in a collateralized economy for loans, it is possible to redefine the payoff function of the agents to obtain the same equilibrium in this new non-collateralized economy. The payoff functions are modified in such a way that they embody some sort of punishment if the agent does not honor at least part of his debt. Conversely, if we have an equilibrium in an economy with utility penalties and we want to implement a system of collateral requirements, it is possible to redefine the payoff functions and initial endowments of the agents to obtain the same equilibrium in the new economy. Lending the collateral and exchanging the financial earnings of the old system for the corresponding in the new system we obtain the modified initial wealth. Also, all these modifications imply the corresponding modification (in utility units) of the payoff function. This is a very natural way to implement a collateral system in a economy where the default is penalized directly in the utility function. The hypotheses used for these results are the concavity of the utility function and the positiveness of the initial endowments. 
Finally, we offer a discussion on the social welfare of these findings. If we pass from an economy with collateral requirements to one with utility penalties, the individual's welfare is maintained. This is a very interesting result because it affirms that both mechanisms used to enforce financial commitments are socially equivalent. In equilibrium the agents achieve the same individual welfare. On the other hand, if we pass from an economy with utility penalties to one with collateral requirements the individual payoff may vary. However, the cost/benefit (in utility units) of implementing the new system equals the variation in the payoff for each individual.

\section{APPENDIX}

In most of the proofs we will use the following version of the Karush-Kuhn-Tucker theorem (see Avriel (1976)). Consider the following maximization problem:

$$
(M P)\left\{\begin{array}{cc}
\text { Maximize } & f(x) \\
\text { subject to } & g_{m}(x) \leq 0 \quad, m=1, \ldots, M \\
& x \in C
\end{array}\right.
$$

where $C$ is a convex set and $f: R^{n} \rightarrow R$ and $g_{m}: R^{n} \rightarrow R$.

Theorem (*): 1) Suppose that $f$ is a concave function and $g_{m}$ is a convex function for each $m$. If $x^{*} \in C$ is the solution of $(M P)$ and there exists $\bar{x} \in C$ such that $g(\bar{x})<0$ (this condition is called Slater's condition), then there exist Lagrange multipliers $\lambda_{m} \geq 0 ; m=1, \ldots, M$ such that for all $x \in C$ we have:

$$
f(x)-f\left(x^{*}\right) \leq \sum_{m} \lambda_{m}\left(g_{m}(x)-g_{m}\left(x^{*}\right)\right)
$$

and the complementary conditions: $\lambda_{m} g_{m}\left(x^{*}\right)=0 ; m=1, \ldots, M$

2) If there exist feasible vectors $x^{*} \in C$ (i.e $g\left(x^{*}\right) \leq 0$ ) and Lagrange multipliers $\lambda_{m} \geq 0 ; m=1, \ldots, M$ such that for all $x \in C$ we have:

$$
f(x)-f\left(x^{*}\right) \leq \sum_{m} \lambda_{m}\left(g_{m}(x)-g_{m}\left(x^{*}\right)\right)
$$


and the complementary conditions: $\lambda_{m} g_{m}\left(x^{*}\right)=0 ; m=1, \ldots, M$, then $x^{*} \in C$ is the solution of $(M P)$.

Proof of Theorem 3.1: The allocation $\left(x^{h}, \theta^{h}, \varphi^{h}\right)$ is optimal for agent $h$ in the economy $\varepsilon_{C}$, then using theorem (*): there exist $\beta^{h} \in R_{+}^{S+1}$ such that for all $x \in R_{+}^{L \times(S+1)}$, $\theta, \varphi \in R_{+}^{J}$,

$$
\begin{aligned}
& u^{h}\left(x_{0}+C \varphi,\left(x_{s}\right)\right)-u^{h}\left(x_{0}^{h}+C \varphi^{h},\left(x_{s}^{h}\right)\right) \leq \beta_{0}^{h}\left\{p_{0}\left(\left(x_{0}+C \varphi\right)-\left(x_{0}^{h}+C \varphi^{h}\right)\right)+\pi\left(\left(\theta-\theta^{h}\right)-\left(\varphi-\varphi^{h}\right)\right)\right\} \\
& +\sum_{s} \beta_{s}^{h}\left\{p_{s}\left(x_{s}-x_{s}^{h}\right)+\sum_{j} d_{s}^{j}\left(\varphi_{j}-\varphi_{j}^{h}\right)-\sum_{j} d_{s}^{j}\left(\theta_{j}-\theta_{j}^{h}\right)-p_{s} Y_{s}\left(\left(x_{0}+C \varphi\right)-\left(x_{0}^{h}+C \varphi^{h}\right)\right)\right\} .
\end{aligned}
$$

Then, for any $\varphi \leq v=\operatorname{Max}\left\{p_{0} w_{0}^{h} /\left(p_{0} C_{j}-\pi_{j}\right), j \in J, h \in H\right\} \underline{1} \quad\left(\right.$ where $\left.\underline{1}=(1, \ldots, 1) \in R^{J}\right)$ and $D_{s}^{j} \in\left[0, p_{s} A_{s}^{j} \varphi_{j}\right]$, we can substitute $x_{0}+C \varphi$ by $x_{0}$ and $d_{s}^{j}=t_{s}^{j} p_{s} A_{s}^{j}$ and rewrite the inequality above as (we will use the $\Delta^{h}$ notation for denoting the deviation from the optimal value):

$$
\begin{aligned}
& u^{h}\left(x_{0},\left(x_{s}\right)\right)-u^{h}\left(x_{0}^{h}+C \varphi^{h},\left(x_{s}^{h}\right)\right) \leq \beta_{0}^{h}\left\{p_{0}\left(x_{0}-\left(x_{0}^{h}+C \varphi^{h}\right)\right)+\pi\left(\Delta^{h} \theta-\Delta^{h} \varphi\right)\right\} \\
& +\sum_{s} \beta_{s}^{h}\left\{p_{s} \Delta^{h} x_{s}+\sum_{j} \Delta^{h} D_{s}^{j}-\sum_{j} t_{s}^{j} p_{s} A_{s}^{j} \Delta^{h} \theta_{j}-p_{s} Y_{s}\left(x_{0}-\left(x_{0}^{h}+C \varphi^{h}\right)\right)\right\} \\
& +\sum_{s, j} \beta_{s}^{h} t_{s}^{j} p_{s} A_{s}^{j} \Delta^{h} \varphi_{j}-\sum_{s, j} \beta_{s}^{h} \Delta^{h} D_{s}^{j} .
\end{aligned}
$$

Then we can define $\tilde{V}^{h}(x, \varphi, D)=u^{h}\left(x_{0},\left(x_{s}\right)\right)-\sum_{s, j} \beta_{s}^{h}\left(t_{s}^{j} p_{s} A_{s}^{j} \varphi_{j}-D_{s}^{j}\right)$ and write down the inequality above as:

$$
\begin{aligned}
& \Delta^{h} \tilde{V}^{h} \leq \beta_{0}^{h}\left\{p_{0}\left(x_{0}-\left(x_{0}^{h}+C \varphi^{h}\right)\right)+\pi\left(\Delta^{h} \theta-\Delta^{h} \varphi\right)\right\} \\
& +\sum_{s} \beta_{s}^{h}\left\{p_{s} \Delta^{h} x_{s}+\sum_{j} \Delta^{h} D_{s}^{j}-\sum_{j} t_{s}^{j} p_{s} A_{s}^{j} \Delta^{h} \theta_{j}-p_{s} Y_{s}\left(x_{0}-\left(x_{0}^{h}+C \varphi^{h}\right)\right)\right\}
\end{aligned}
$$

In the economy $\varepsilon_{C}$, the complementary conditions for the agent $h$ maximization problem are: 


$$
\begin{gathered}
\beta_{0}^{h}\left\{p_{0}\left(x_{0}^{h}-w_{0}^{h}\right)+\left(p_{0} C-\pi\right) \varphi^{h}+\pi \theta^{h}\right\}=0 \\
\beta_{s}^{h}\left\{p_{s}\left(x_{s}^{h}-w_{s}^{h}\right)+\sum d_{s}^{j} \varphi_{j}^{h}-\sum d_{s}^{j} \theta_{j}^{h}-p_{s} Y_{s}\left(x_{0}^{h}+C \varphi^{h}\right)\right\}=0
\end{gathered}
$$

If we substitute $D_{s}^{h j}=d_{s}^{j} \varphi^{h}$ and $d_{s}^{j} \theta_{j}^{h}=t_{s}^{j} p_{s} A_{s}^{j} \theta_{j} \quad$ the complementary conditions for agent $h$ in the economy $\varepsilon_{P}$ will result. Theorem (*) shows the optimality of the allocation for the new payoff function $\tilde{V}^{h}$ in the economy $\varepsilon_{P}$.

The market clear conditions are easily checked.

Proof of Corollary 3.2: In this case the payment rate is the same in both economies, i.e. $d_{s}^{j}=t_{s}^{j} p_{s} A_{s}^{j}$ and $D_{s}^{h j}=d_{s}^{j} \varphi_{j}^{h}$. Let us substitute the equilibrium allocation in the new payoff function defined in the proof of theorem 3.1:

$$
\begin{aligned}
\tilde{V}^{h}\left(x_{0}^{h}+C \varphi,\left(x_{s}^{h}\right), \varphi^{h}, D^{h}\right) & =u^{h}\left(x_{0}^{h}+C \varphi^{h},\left(x_{s}^{h}\right)\right)-\sum_{s, j} \beta_{s}^{h}\left(t_{s}^{j} p_{s} A_{s}^{j} \varphi_{j}^{h}-D_{s}^{h j}\right) \\
& =u^{h}\left(x_{0}^{h}+C \varphi^{h},\left(x_{s}^{h}\right)\right)-\sum_{s, j} \beta_{s}^{h}\left(d_{s}^{j} \varphi_{j}^{h}-d_{s}^{j} \varphi_{j}^{h}\right) \\
& =u^{h}\left(x_{0}^{h}+C \varphi^{h},\left(x_{s}^{h}\right)\right) .
\end{aligned}
$$

Proof of Theorems 3.3 and 3.4: By using the theorem (*), the optimal allocation $\left(x^{h}, \theta^{h}, \varphi^{h}, D^{h}\right)$ of agent $h$ in the economy $\varepsilon_{P}$ must satisfy the following: there exists $\alpha^{h} \in R_{+}^{S+1}$ such that for all $(x, \theta, \varphi, D) \in R_{+}^{L(S+1)} \times R_{+}^{J} \times R_{+}^{J} \times R_{+}^{S J}$ we have:

$$
\begin{aligned}
\Delta^{h} u \leq & \sum_{s, j} \lambda_{s}^{j} \Delta^{h}\left[p_{s} A_{s}^{j} \varphi_{j}-D_{s}^{j}\right]+\alpha_{0}^{h}\left\{p_{0} \Delta^{h} x_{0}+\pi\left(\Delta^{h} \theta-\Delta^{h} \varphi\right)\right\} \\
& +\sum_{s} \alpha_{s}^{h}\left\{p_{s} \Delta^{h} x_{s}+\sum_{j} \Delta^{h} D_{s}^{j}-\sum_{j} t_{s}^{j} p_{s} A_{s}^{j} \Delta^{h} \theta_{j}-p_{s} Y_{s} \Delta^{h} x_{0}\right\},
\end{aligned}
$$

where $\Delta^{h}$ represents the variation with respect to the optimal value. If we define the function:

$$
\begin{aligned}
\hat{V}^{h}(x, \theta, \varphi, D)= & u^{h}(x)-\sum_{s, j} \lambda_{s}^{j}\left[p_{s} A_{s}^{j} \varphi_{j}-D_{s}^{j}\right]^{+}+\alpha_{0}^{h}\left\{p_{0}-\sum_{s}\left(\frac{\alpha_{s}^{h}}{\alpha_{0}^{h}}\right) p_{s}\right\} C \varphi \\
& +\sum_{s, j} \alpha_{s}^{h}\left(t_{s}^{j} p_{s} A_{s}^{j} \varphi_{j}-D_{s}^{j}\right)-\sum_{s, j} \alpha_{s}^{h} d_{s}^{j}\left(\theta_{j}-\varphi_{j}\right),
\end{aligned}
$$

and substitute it in the inequality above, the result will be: 


$$
\hat{V}^{h}(x, \theta, \varphi, D)-\hat{V}^{h}\left(x^{h}, \theta^{h}, \varphi^{h}, D^{h}\right) \leq \alpha_{0}^{h} L_{0}+\sum_{s} \alpha_{s}^{h} L_{s}
$$

where $\quad L_{0}=p_{0} \Delta^{h}\left(x_{0}+C \varphi\right)-\pi\left(\Delta^{h} \theta-\Delta^{h} \varphi\right) \quad$ and $\quad L_{s}=p_{s} \Delta^{h} x+\sum_{j} d_{s}^{j} \Delta^{h}\left(\varphi_{j}-\theta_{j}\right)$ $-p_{s} Y_{s} \Delta^{h}\left(x_{0}+C \varphi\right)$. To eliminate the variable $D$ from this inequality (because it is not a decision variable for the individual problem in a collateralized economy) it is sufficient to consider a $\varphi-D$ path which contains $\left(\varphi_{j}^{h}, D_{s}^{h j}\right)$ in its graph. Using the $\phi_{s n}^{h j}$ function defined before Theorem 3.4, we consider the path $D_{s}^{j}=p_{s} A_{s}^{j} \varphi_{j}-\phi_{s n}^{h j}\left(\varphi_{j}\right)$. Substituting these paths into the function $\hat{V}^{h}$ we will obtain the following payoff function:

$$
\begin{aligned}
\tilde{V}_{n}^{h}(x, \varphi, \theta)= & u^{h}(x)+\sum_{s, j} \alpha_{s}^{h}\left(t_{s}^{j} p_{s} A_{s}^{j} \theta_{j}-p_{s} A_{s}^{j} \varphi_{j}\right)-\tilde{R}^{h}(\theta, \varphi)+\alpha_{0}^{h}\left[p_{0}-\sum_{s \in S} \frac{\alpha_{s}^{h}}{\alpha_{0}^{h}} p_{s} Y_{s}\right] C \varphi \\
& +\sum_{s, j}\left(\alpha_{s}^{h}-\lambda_{s}^{j}\right) \phi_{s n}^{h j}\left(\varphi_{j}\right) .
\end{aligned}
$$

which is exactly the payoff function of Theorem 3.4. To obtain the corresponding payoff function of Theorem 3.3 we have two cases:

1o.) If $D_{s}^{h j}=p_{s} A_{s}^{j} \varphi_{j}^{h}>0$ then $\phi_{s n}^{h j} \equiv 0$,

2o.) If $D_{s}^{h j} \in\left(0, p_{s} A_{s}^{j} \varphi_{j}^{h}\right)$ then $\alpha_{s}^{h}=\lambda_{s}^{j}$.

This implies that $\phi_{s n}^{h j} \equiv 0$. Therefore if $D^{h} \gg>0$ then the payoff function of Theorem 3.3 results.

The complementary conditions are easily checked. So we have the optimality of $\left(x^{h}, \theta^{h}, \varphi^{h}\right)$ on the budget constraint of individual $h$ with payoff function $\tilde{V}_{n}^{h}$ in the economy with collateral system $C$.

The proof of the market clear conditions for the first period is straightforward. For the second period we need to define the set $J_{s}=\left\{j \in J / p_{s} A_{s}^{j} \leq p_{s} Y_{s} C_{j}\right\}$ (the set of all assets whose promises are honored in state $s$ ). Then, the initial endowment of agent $h$ in state $s$ can be written as:

$$
\tilde{w}_{s}^{h}=w_{s}^{h}+\sum_{j} A_{s}^{j}\left(t_{s}^{j} \theta_{j}^{h}-\rho_{s}^{h j} \varphi_{j}^{h}\right)-\sum_{j \in J_{s}} A_{s}^{j}\left(\theta_{j}^{h}-\varphi_{j}^{h}\right)-\sum_{j \notin J_{s}} Y_{s} C_{j}\left(\theta_{j}^{h}-\varphi_{j}^{h}\right)-Y_{s} C \varphi^{h} .
$$


Then, from the market clear conditions of the second period of the economy $\varepsilon_{P}$ and the following identity: $t_{s}^{j}=\frac{\sum_{h} \rho_{s}^{h j} \varphi_{j}^{h}}{\sum_{h} \varphi_{j}^{h}}$, we obtain the market clear conditions of the second period for the economy $\varepsilon_{C}$.

\section{References}

Araujo A., Páscoa M. and Torres-Martinez J., "Collateral Avoids Ponzi Schemes in Imcomplete Markets", Econometrica, Vol. 70, No. 4, (2002), pp. 1613-1638.

Avriel M., Nonlinear programming - Analysis and Methods. Englewood Cliff, N. J.: Prentice-Hall, Inc. (1976).

Dubey P., Geanakoplos J. and Shubik M., "Default and punishment in general equilibrium", Econometrica, Vol. 73 - 1, (2005), 1 - 37.

Geanakoplos J. and Zame W., "Collateral and the Enforcement of Intertemporal Contracts", Yale University Working Paper (2002).

Goodhart Ch., Sunirand P. and Tsomocos D., "A Model to Analyse Financial Fragility", Oxford Financial Research Centre Working Paper No. 2003fe13. (2003a).

Goodhart Ch., Sunirand P. and Tsomocos D., "A Model to Analyse Financial Fragility: Applications”, Mimeo Bank of England. (2003b).

Kubler, F., Schmedders, K., "Stationary Equilibria in Asset-Pricing Models with Incomplete Markets and Collateral', Econometrica, 71(6), (2003), pp.1767-1793. 
Orrillo, J., "Making Promises in Infinite-Horizon Economies with Default", Catholic University of Brasilia Working Paper, (2002).

Zame W., "Efficiency and the Role of Default when Security Markets are Incomplete", American Economic Review 83 (1993), 1142-1164. 